\title{
Down-regulation of the apoptosis-inducing factor or Bcl-2 inhibitor of transcription by RNA interference can alleviate TAp63 $\gamma$-induced apoptosis in esophageal squamous carcinoma EC9706 cells
}

\author{
TIANLI FAN ${ }^{1}$, GUOZHONG JIANG ${ }^{2}$, ZHENHE SUO ${ }^{3}$, HONGTAO LIU $^{4}$, PEIRONG XU ${ }^{4}$, \\ ZHENYU JI $^{5}$, LIRONG ZHANG ${ }^{1}$ and GUANRUI YANG ${ }^{5}$
}

\begin{abstract}
${ }^{1}$ Department of Pharmacology, School of Medicine, Zhengzhou University; ${ }^{2}$ Department of Pathology, The First Affiliated Hospital of Zhengzhou University, ZhengZhou 450052, P.R. China; ${ }^{3}$ The National Hospital-The Norwegian Radium Hospital Medical Center, University of Oslo, Montebello, N-0310, Oslo, Norway; ${ }^{4}$ Department of Biology Engineering, Zhengzhou University; ${ }^{5}$ Henan Institute of Medical Science, Zhengzhou University, ZhengZhou 450052, P.R. China
\end{abstract}

Received March 16, 2009; Accepted May 25, 2009

DOI: 10.3892/ijo_00000347

\begin{abstract}
In this communication, the roles of apoptosisinducing factor (AIF) and Bcl-2 inhibitor of transcription (Bit1) in TAp63 $\gamma$-induced apoptosis were investigated in human esophageal squamous cancer EC9706 cells. Positive RNA and protein expressions of AIF and Bit1 in these cells were verified. However, no TAp63 $\gamma$ could be detected. Transfection of expression vector pcDNA3.1-TAp63 $\gamma$ into EC9706 resulted in TAp63 $\gamma$ expression, and peak level apoptosis was observed $24 \mathrm{~h}$ after the transfection disclosed by DNA fragmentation assay. In addition, it was found with Western blot that AIF and Bit1 were released into cytosol from mitochondria, and AIF was further translocated into nucleus, during the stage of TAp63 $\gamma$-induced cell apoptosis. Down-regulation of either AIF or Bit1 by RNA interference could, however, alleviate TAp63 $\gamma$-induced cell apoptosis. In conclusion, TAp63 $\gamma$ could induce apoptosis in human esophageal squamous cancer EC9706 cells, through at least releasing AIF and Bit1 from mitochindria into cytosol and nucleus, where apoptotic cascade takes place. These findings indicate that mitochondria-released proapoptotic proteins, AIF and Bit1, are important factors in a TAp63 $\gamma$-induced EC9706 cell apoptosis pathway.
\end{abstract}

\section{Introduction}

p63 is a member of the p53 family $(1,2)$. It contains regions of homology to p53 and shares $60 \%$ sequence identity with

Correspondence to: Professor Guanrui Yang, Henan Institute of Medical Science, Zhengzhou University, 40 Daxue Road, Zhengzhou, Henan 450052, P.R. China

E-mail: guanrui_yang@sina.com

Key words: apoptosis, TAp63, AIF, Bit1, esophagus the DNA-binding domain, $22 \%$ identity with the N-terminal transactivation domain and $37 \%$ identity with the oligomerization domain of p53. In contrast to p53, however, human p63 has two promoters, which result in two different types of protein products with opposite functions: the full-length transcriptionally active TAp63 and the dominant-negative $\Delta$ Np63. Furthermore, p63 can vary at its carboxy-terminal end, as a result of alternative splicing at the 3 ' portion of the mRNA, which yields isoforms such as $\alpha, \beta$ and $\gamma$ (3).

p63 shares some of p53 functions due to the strong structural similarity (3). There is mounting evidence indicating that a large number of endogenous p53 target genes are transactivated by 663 to mediate cell cycle arrest and induce apoptosis, independently of p53 status (3-5). In addition, Perp, which originally was identified as an apoptosisassociated target of the p53 tumor suppressor $(6,7)$, is the direct target of p63 clearly involved in mediating developmental program in vivo $(8,9)$. Recent evidence has revealed that cytoplasmic p53 directly activates Bcl-2 proteins to initiate apoptosis, but nuclear p53 regulates the expression of proapoptotic genes $(10,11)$, such as the PUMA gene. In contrast, TAp63 $\gamma$ functions only in the nucleus showing strong proapoptotic ability (3).

In stressed cells, the induction of cytosolic Bax protein expression may contribute to its activation, oligomerization and translocation to mitochondria, triggering loss of the mitochondrial membrane potential and the release of cyto-c and apoptosis-inducing factor (AIF) $(8,12,13)$. Despite the mechanisms involving p53 mediated apoptosis pathways, it is still suggested that at least some signaling pathways are utilized by other members of p53 family in the absence of p53 (14).

AIF and Bcl-2 inhibitor of transcription (Bit1) are both mitochondrial proteins, and are recently identified caspaseindependent apoptosis effectors. AIF can translocate to the nucleus to execute chromatin condensation and apoptosis by a mechanism independent of caspases (15). Many studies 
demonstrate that the release of AIF can be regulated by the Bcl-2 protein family (16-20). Bit1, a proapoptotic protein whose cytoplasmic expression induces cell death, interacts with AES (a member of the Groucho family of transcriptional corepressors) to induce apoptosis (21). This type of apoptosis is also called anoikis which means loss of cell attachment to the extracellular matrix to cause apoptosis (21). However, cell attachment to fibronectin counteracts the apoptotic effect of Bit1, and overexpression of the antiapoptotic family member Bcl-2 or Bcl-xl prevents the release of Bit1. Staurosporine treatment results in the release of Bit 1 into the cytosol (21). Similarly, staurosporine also induces mitochondrial permeability and the release of AIF. However, the changes of mitochondrial permeability and the apoptotic targets activated by p63 remain to be discovered.

To clarify the role of molecular mechanism of TAp63 in apoptosis, we chose to investigate the correlation between TAp63 expression and the release of AIF and Bit1 from mitochondria in vitro, by introducing the TAp63 $\gamma$ expression vector into human esophageal squamous carcinoma EC9706 cells. TAp63 $\gamma$ was chosen in this study because it has been proved to be the most powerful transactivating TAp63 isoform (3).

\section{Materials and methods}

Cells, vectors, antibodies and reagents. Human esophageal squamous carcinoma cell line (EC9706) was kindly provided by State Key Laboratory of Molecular Oncology, Chinese Academy of Medical Science; pcDNA3.1, pcDNA3.1TAp63 $\gamma$, pcDNA3.1-p53 and pcDNA3.1/Myc-His-TAp63 $\gamma$ were kindly provided by Dr Yaohe Wang; chicken polyclonal IgY to CGI-147(Bit1) and HRP-linked affinity-purified rabbit anti-IgY were obtained from Genway; goat polyclonal IgG to AIF, mouse polyclonal to $\mathrm{p} 53$, rabbit polyclonal to $B$-actin and HRP-linked antibodies were purchased from Santa Cruz; mouse monoclonal $\mathrm{IgG}_{1}$ to his-tag was from Novagen. Rabbit polyclonal IgG to histone $\mathrm{H}_{3}$ and voltage-dependent anion channel (VDAC) were bought, respectively, from Millipore and Rockland; RPMI-1640 medium was from Gibco; one step reverse transcriptase-polymerase chain reaction (RT-PCR) kit was from Takara; DNA Ladder kit, TdT-mediated dUTP nick-end labeling (TUNEL) apoptosis detection kit and Annexin V-FITC kit were from Beyotime Biotechnology, KeyGen Biotechnology and Beckman Coulter, respectively; mitochondrial/cytosol fractionation kits were purchased from BioVision; NE-PER nuclear and cytoplasmic extraction reagents were obtained from Pierce Biotechnology; TransIT-LT1 transfection reagent was purchased from Mirus; oligonucleotides and the primers were obtained from Shanghai Sangon Biological Engineering technology and service Co., Ltd.

Cell culture. EC9706 was cultured in RPMI-1640 medium containing $10 \%$ fetal bovine serum, $100 \mathrm{U} / \mathrm{ml}$ penicillin and streptomycin, in a humidified atmosphere of $5 \% \mathrm{CO}_{2}$ at $37^{\circ} \mathrm{C}$.

$R N A$ preparation and $R T-P C R$. Total RNA was prepared from the EC9706 cells with TRIzol reagent according to the manu- facturer's protocol and treated with the DNA-free kit to remove residual genomic DNA. Briefly, the RNA isolated ( $1 \mu \mathrm{g})$ was used as template to perform one step RT-PCR according to the protocol and $\beta$-actin or glyceraldehyde-3-phosphate dehydrogenase (GAPDH) was used as an internal control. The following oligonucleotide primers were used for analysis of AES, Bit1, TAp63 $\gamma$, TAp63 and $\triangle$ Np63 amplification. For AES (508 bp), forward 5'-GCGACCGCATCAAAGAC-3'; reverse 5'-TTCTCGCCATCATCCTCC-3'; for Bit1 (428 bp), forward 5'-ATGCCCTCCAAATCCTT-3'; reverse 5'ATTAAACTTACAGTCAGTCCC-3'; for TAp63 $\gamma$ (1346 bp), forward 5'-ATGTCCCAGAGCACACAGAC-3'; reverse 5'TATGGGTACACTGATCGGTT-3'; for $\triangle \mathrm{Np} 63$ (335 bp), forward 5'-TGCCCAGACTCAATTTAGTGAG-3'; reverse 5'-TCTGGATGGGGCATGTCTTTGC-3'; for TAp63 (334 bp), forward 5'-GACCTGAGTGACCCCATGTG-3'; reverse 5'-TCTGGATGGGGCATGTCTTTGC-3'; for ß-actin (315 bp), forward, 5'-TCCTGTGGCATCCACGAAACT-3'; reverse 5'GAAGCATTTGCGGTGGACGAT-3'; for GAPDH (915 bp), forward 5'-AAGGTCGGAGTCAACGGATTTG-3'; reverse 5'-CTTGACAAAGTGGTCGTTGAGG-3'. The amplified products were subjected to electrophoresis on $1 \%$ agarose gels.

TransIT-LT1 transfection. Approximately $24 \mathrm{~h}$ prior to transfection, cells were plated at a density of $2-6 \times 10^{5}$ cells in complete growth medium to obtain $50-70 \%$ confluence. The following day, $15 \mu \mathrm{l}$ of TransIT-LT1 transfection reagent (2-8 $\mu \mathrm{l} / 1 \mu \mathrm{g}$ DNA) was directly added into $500 \mu \mathrm{l}$ of serumfree medium and mixed completely by gentle pipetting and incubated at RT for $20 \mathrm{~min}$. Then, $5 \mu \mathrm{g}$ of plasmid DNA was added to the diluted TransIT-LT1 transfection reagent and mixed by gentle pipetting. The mixture was incubated at RT for $30 \mathrm{~min}$. The medium was replaced with $3 \mathrm{ml}$ of fresh complete growth medium per dish. Finally, the TransIT-LT1 reagent/DNA complex mixture was added to the dishes. The dishes were kept gently rocking for a few minutes in order to distribute the complex evenly, and incubated for $24 \mathrm{~h}$. After that, cells were collected for the following experiments.

DNA fragmentation assay. Cells were transfected with pcDNA3.1, pcDNA3.1-TAp63 $\gamma$ and pcDNA3.1-p53 vectors, respectively and harvested at $6,12,24$ and $48 \mathrm{~h}$ after the transfection. Cells were rinsed with PBS and incubated with lysate buffer containing protease $\mathrm{K}$ at $50^{\circ} \mathrm{C}$ overnight. DNA fragments were purified by phenol-chloroform extraction and ethanol precipitation at $-70^{\circ} \mathrm{C}$, and dissolved in $50 \mu \mathrm{l}$ of TE. Purified DNA $(3 \mu \mathrm{l})$ from each sample was loaded into $1 \%$ agarose gel, electrophoresed for $30 \mathrm{~min}$ at 75 volts and visualized under ultraviolet light after ethidium bromide staining.

TUNEL assay. Cells were transfected with pcDNA3.1 and pcDNA3.1-TAp63 $\gamma$ vectors and harvested at $24 \mathrm{~h}$, respectively. After rinsing with PBS, $1 \times 10^{4}$ cells were plated on polylysinecoated slides. Air dried cell samples were fixed with a freshly prepared fixation solution for $1 \mathrm{~h}$ at $15-25^{\circ} \mathrm{C}$ and then rinsed with PBS three times. After incubated with blocking solution for $10 \mathrm{~min}$ at $15-25^{\circ} \mathrm{C}$ and rinsed with PBS three times, slides were incubated in permeabilisation solution for $2 \mathrm{~min}$ on ice. 
The samples were rinsed twice with PBS and then kept dry. To each slide $50 \mu 1$ TUNEL reaction mixture was added and incubated for $60 \mathrm{~min}$ at $37^{\circ} \mathrm{C}$ in wet and dark atmosphere. Rinsed and kept dry with filter paper, $50 \mu 1$ Streptavidin-HRP working solution was added and incubated for $30 \mathrm{~min}$ at $37^{\circ} \mathrm{C}$ in wet and dark atmosphere. After rinsing and drying, $50 \mu 1$ DAB substrate was added and incubated for $10 \mathrm{~min}$ at 15 $25^{\circ} \mathrm{C}$. Finally, samples were rinsed three times and mounted under glass coverslip and analyzed with light microscope. Negative control (label solution contained no terminal deoxynucleotidyl transferase in TUNEL reaction mixture) and positive control (incubation of permeabilized cells with $100 \mu \mathrm{l}$ DNAse 1 buffer for $10 \mathrm{~min}$ at $15-25^{\circ} \mathrm{C}$, prior to labeling procedures) were also performed.

Annexin V-FITC kit apoptosis assay. The cultured cells were harvested after the $24 \mathrm{~h}$ transfection, with pcDNA3.1 and pcDNA3.1-TAp63 $\gamma$, respectively, washed with ice-cold PBS and centrifuged for $5 \mathrm{~min}$ at $500 \mathrm{xg}$ at $4^{\circ} \mathrm{C}$. The supernatant was discarded, and the cell pellet was resuspended in ice-cold binding buffer. Annexin V-FITC solution $(5 \mu \mathrm{l})$ and $2.5 \mu \mathrm{l}$ of PI was added to $100 \mu \mathrm{l}$ of the cell suspension and then kept on ice for $10 \mathrm{~min}$-incubation in dark before $400 \mu \mathrm{l}$ of ice-cold binding buffer was added to the preparation. The suspension was gently mixed before it was analyzed within $30 \mathrm{~min}$ by flow cytometry.

RNA interference (RNAi). Hairpin oligonucleotides of AIF and Bit1 were respectively designed according to Yuan et al (22) and Jan et al (21). For AIF, the sense sequence is 5'-GATC CGCTTGTTCCAGCGATGGCATTTCAAGAGAATGCC ATCGCTGGAACAAGTTTTTTGGAAA-3'; the antisense sequence is 5'-AGCTTTTCCAAAAAACTTGTTCCAGC GATGGCATTCTCTTGAAATGCCATCGCTGGAACAA GCG-3'. For Bit1, the sense sequence is 5'-GATCCGCTT GGTTATGGAATATTTGTTCAAGAGACAAATATTCC ATAACCAAGTTTTTTGGAAA-3'; the antisence sequence is 5'-AGCTTTTCCAAAAAACTTGGTTATGGAATATTT GTCTCTTGAACAAATATTCCATAACCAAGCG-3. For negative control, the sense sequence is 5'-GATCCGTTCTC CGAACGTGTCACGTTTCAAGAGAACGTGACACGTT CGGAGAATTTTTTGGAAA-3'; the antisense sequence is 5'-AGCTTTTCCAAAAAATTCTCCGAACGTGTCACGT TCTCTTGAAACGTGACACGTTCGGAGAACG-3'. The sense strand and anti-sense one were annealed to form hairpin small interfence RNA (siRNA) template insert. The annealed siRNA template was ligated with pSilencer3.1-H1-neo using T4 DNA ligase, transformed into competent Escherichia coli DH5 $\alpha$ and amplified. Clones with the hairpin siRNA insert were identified by digesting isolated plasmid DNA with BamHI and HindIII and the inserted sequence was verified by sequencing. Then, pSilencer3.1-H1-neo containing the siRNA templates was transfected into EC9706 cells. After G418 selection, AIF and Bit1 protein expression in the experimental and control groups of EC9706 cells were analyzed with Western blot as described above.

Mitochondrial/cytosol fractionation. Twenty-four hours after transfection, respectively, with pcDNA3.1-TAp63 $\gamma$, pcDNA3.1-p53 (positive control) and pcDNA3.1 (negative control), cells $\left(5 \times 10^{7}\right)$ were harvested and rinsed with ice-cold PBS, and then centrifuged at $600 \mathrm{x}$ g for $5 \mathrm{~min}$ at $4^{\circ} \mathrm{C}$. The cells were resuspended with $1 \mathrm{ml}$ of the cytosol extraction buffer mix containing DL-Dithiothreitol and protease inhibitors, incubated on ice for $10 \mathrm{~min}$, and homogenized with an ice-cold tissue grinder on ice for $30-50$ passages. The homogenate was transferred to a $1.5 \mathrm{ml}$ microcentrifuge tube and centrifuged at $700 \mathrm{x}$ g for $10 \mathrm{~min}$ at $4^{\circ} \mathrm{C}$. The supernatant was collected into a fresh $1.5 \mathrm{ml}$ tube and centrifuged at $10,000 \mathrm{x} \mathrm{g}$ for $30 \mathrm{~min}$ at $4^{\circ} \mathrm{C}$. The supernatant was collected as cytosolic fraction, and the pellet was resuspend with $100 \mu \mathrm{l}$ of mitochondrial extraction buffer mix containing DTT and protease inhibitors, and vortexed for $10 \mathrm{sec}$ as mitochondrial fraction.

Nuclear protein extraction. Twenty-four hours after transfection, respectively, with pcDNA3.1-TAp63 $\gamma$, pcDNA3.1-p53 (positive control) and pcDNA3.1 (negative control), cells $\left(5 \times 10^{7}\right)$ were harvested and rinsed with ice-cold PBS, and then centrifuged at $600 \times \mathrm{g}$ for $5 \mathrm{~min}$ at $4^{\circ} \mathrm{C}$. The cell pellet was resuspended with $200 \mu \mathrm{l}$ of ice-cold CER I, vortexed and incubated on ice for $10 \mathrm{~min}$ before adding $11 \mu \mathrm{l}$ of ice-cold CER I, then vortexed and incubated on ice for $1 \mathrm{~min}$ before it was centrifuged at $16,000 \mathrm{x}$ g for $5 \mathrm{~min}$ at $4^{\circ} \mathrm{C}$. The sedimentation was resuspended with $50 \mu \mathrm{l}$ of ice-cold NER, vortexed on the highest setting for $15 \mathrm{sec}$, kept on ice and vortexed for $15 \mathrm{sec}$ every $10 \mathrm{~min}$, for a total of $40 \mathrm{~min}$ before it was centrifuged at $16,000 \mathrm{x}$ g for $10 \mathrm{~min}$ at $4^{\circ} \mathrm{C}$. The supernatant fractionation was immediately transferred to a clean pre-chilled tube and stored at $-80^{\circ} \mathrm{C}$ for use.

Western blot. Subcellular protein $(50 \mu \mathrm{g})$ was added to $2 \mathrm{x}$ protein sample buffer, heated at $100^{\circ} \mathrm{C}$ for 5 min and separated with sodium dodecyl sulfated-polyacrylamide electrophoresis gels (SDS-PAGE) with $20 \mu \mathrm{l}$ of pre-stained protein molecular weight marker as standard. The SDS-PAGE separated proteins were electro-transferred to nitrocellulose membranes (Hybond-C pure, Amersham) in transfer buffer containing $25 \mathrm{mM}$ Tris, $193 \mathrm{mM}$ glycine and $20 \%$ methanol. The membranes loaded with the proteins were treated with $5 \%$ non-fat milk in $0.05 \%$ Tween of Tris-buffered saline (TBS-T) at RT for $1 \mathrm{~h}$ and then rinsed three times and incubated with anti-AIF or anti-Bit1 antibodies diluted in $0.5 \%$ non-fat milk, respectively, at RT for $2 \mathrm{~h}$. The blots were rinsed three times in TBS-T and incubated with a 1:5000 diluted rabbit anti-goat secondary antibody for anti-AIF antibody or a rabbit antichicken secondary antibody for anti-Bit1 antibody conjugated to horseradish peroxidase for $1 \mathrm{~h}$ at $\mathrm{RT}$ before extensively washed with TBS-T. The blots were developed with ECL reagents (Amersham) according to the manufacturer's instructions and photographed.

\section{Results}

Expression of AIF, Bit1, p63 and p53 in EC9706 cells. Expression of these factors in EC9706 cells were examined with the methods of RT-PCR and Western blot. Both Bit1 and AES expressed at mRNA level were disclosed with RTPCR (Fig. 1A). Positive protein expressions of AIF and Bit1 were observed only in the mitochondria of the cells with the 


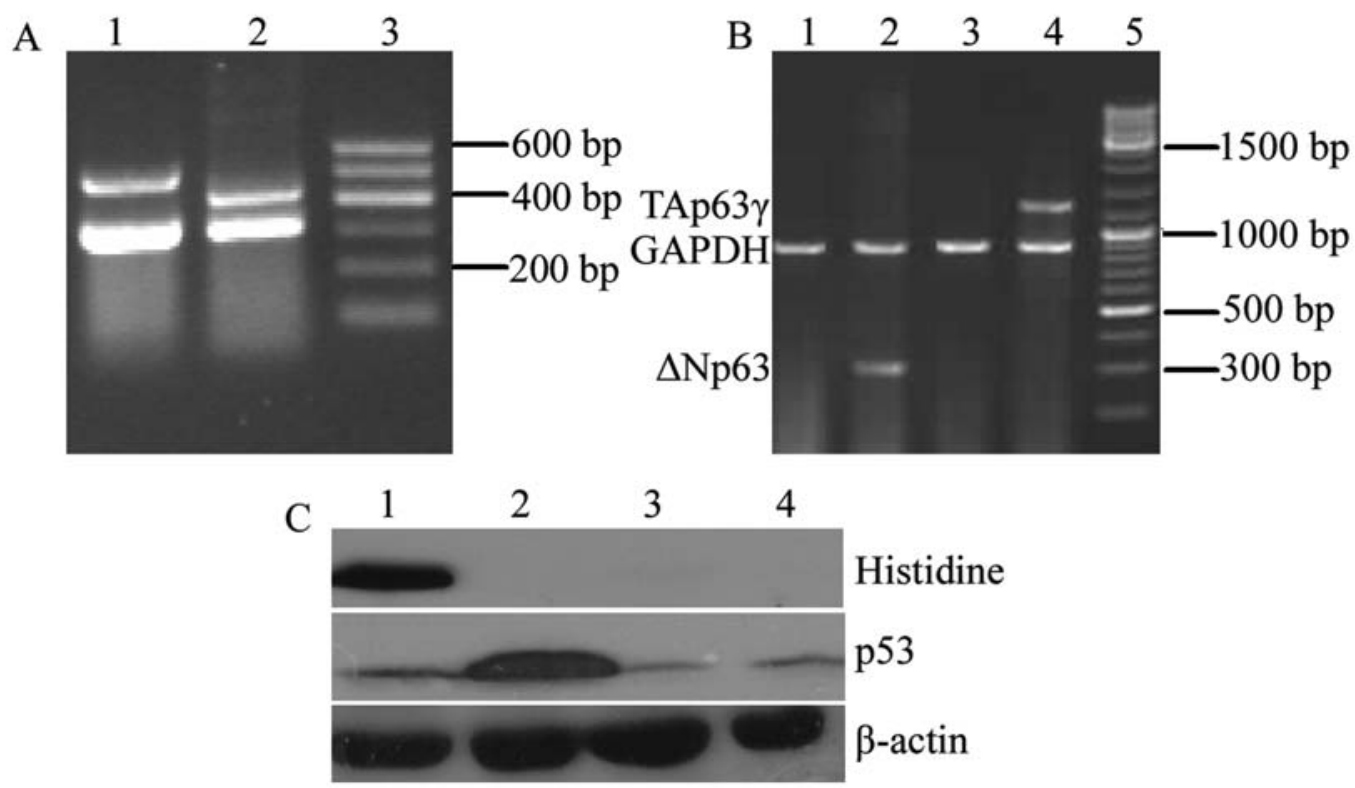

Figure 1. Expression of AIF, Bit1, p63 and p53 in EC9706 cells. (A) Expression of Bit1 (428 bp) and AES (508 bp) in EC9706 cells by RT-PCR. 1, B-actin

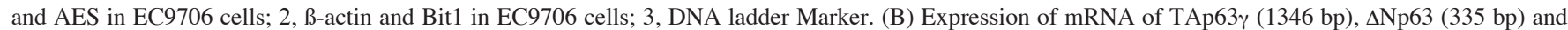
TAp63 (334 bp) indifferent groups by RT-PCR. 1, TAp63 in untreated group; 2, $\Delta$ Np63 in untreated group; 3, TAp63 $\gamma$ in pcDNA3.1 transfection group; 4 , TAp63 $\gamma$ in pcDNA3.1-TAp63 $\gamma$ transfection group; 5, DNA ladder Marker. (C) Western blot with different protein levels $24 \mathrm{~h}$ after transfection. 1, pcDNA3.1/Myc-His-TAp63 $\gamma$ transfection group; 2, pcDNA3.1-p53 transfection group; 3, pcDNA3.1 transfection group; 4, untreated group.

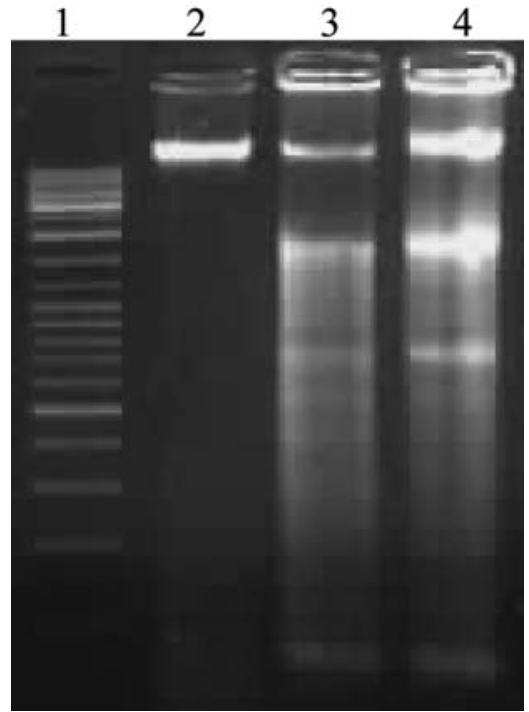

Figure 2. DNA fragmentation assay of DNA isolated $24 \mathrm{~h}$ after the EC9706 cells were respectively transfected with pcDNA3.1, pcDNA3.1-TAp63 $\gamma$ and pcDNA3.1-p53. 1, DNA ladder Marker; 2, pcDNA3.1 transfection group; 3, pcDNA3.1-TAp63 $\gamma$ transfection group; 4, pcDNA3.1-p53 transfection group.

Western blot (Fig. 3B-a). RT-PCR verified the sub-type of p63 in EC9706 cells is $\Delta$ Np63 (Fig. 1B-2), but did not reveal any TAp63 expression in these cells (Fig. 1B-1). Cells transfected with pcDNA3.1-TAp63 $\gamma$ expressed TAp63 $\gamma$ in mRNA levels (Fig. 1B-4), while cells transfected with pcDNA3.1 did not express TAp63 $\gamma$ (Fig. 1B-3). Before and after pcDNA3.1/ Myc-His-TAp63 $\gamma$ or pcDNA3.1-p53 transfection, a Western blot with protein levels of TAp63 $\gamma$ or p53 was performed. The results showed that cells expressed highly TAp63 $\gamma$ and p53 in protein levels after 24-h transfection, however, they did not express any TAp63 $\gamma$ or expressed p53 at a lower level before transfection (Fig. 1C).

TAp63r induces EC9706 cell apoptosis, but cannot be completely blocked by the pan-caspase inhibitor z-VAD-fmk. Agarose gel electrophoresis revealed a DNA ladder, a typical feature of apoptosis in both the TAp63 $\gamma$-transfected cells and the p53-transfected cells, but not in the pcDNA3.1transfected cells (Fig. 2). DNA fragmentation reached a peak level $24 \mathrm{~h}$ after TAp63 $\gamma$ transfection (data not shown), so transfection at $24 \mathrm{~h}$ was chosen for all the experiments. TUNEL assay also showed that the number of positive apoptotic cells $(239 / 1500)$ in TAp63 $\gamma$-transfection group was much more than that $(34 / 1500)$ in pcDNA3.1-transfection group (Table I). To further analyse the relationships between TAp63 $\gamma$ expression and apoptosis, flow cytometry was performed. It was found that the percentages of Annexin Vpositive cells were $1.37 \%$ in the negative control (Fig. 6A-a) and $13.64 \%$ in the TAp63 $\gamma$-transfected cells (Fig. 6A-b). Later stage apoptosis, revealed by both Annexin $\mathrm{V}$ and propidium iodide (PI) uptake (upper right panels), was also greater in the TAp63 $\gamma$-transfected cells compared with that in the negative control ( 9.63 vs. $4.49 \%)$, indicating that expression of TAp63 $\gamma$ induces apoptosis in EC9706 cells.

To study the role of caspase in TAp63 $\gamma$-induced apoptosis, cells were pretreated with pan-caspase inhibitor z-VAD-fmk $(50 \mu \mathrm{M}) 30 \mathrm{~min}$ before TAp63 $\gamma$-transfection. However, it showed only $4.03 \%$ of cells with apoptotic changes and $5.12 \%$ of cells with later stage apoptosis changes (Fig. 6A-c), suggesting that TAp63 $\gamma$-induced apoptosis could be 


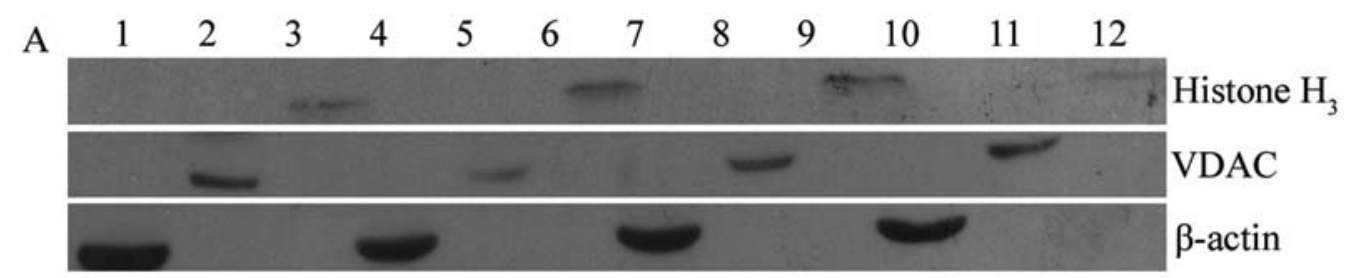

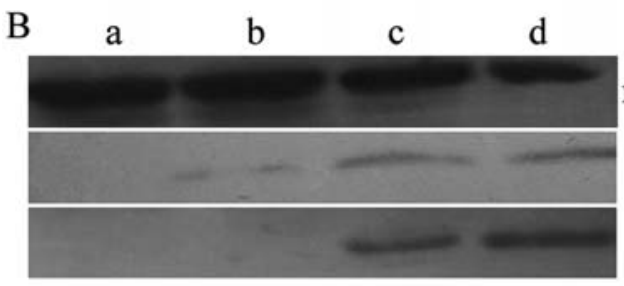

AIF

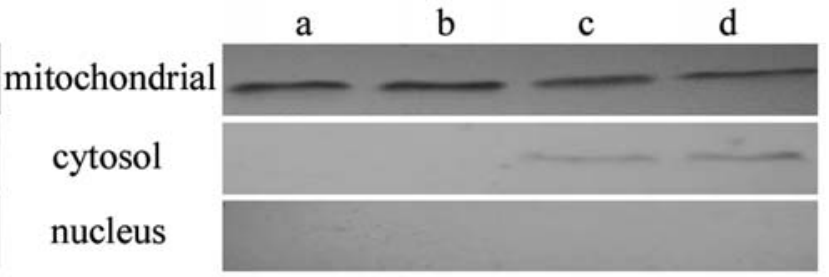

Bit1

Figure 3. Translocation of AIF and Bit1. (A) Detection of the identity and purity of the nucleus, mitochondrial and cytosol (non-mitochondria) proteins. 1, 2 and 3, untreated group; 4, 5 and 6, pcDNA3.1 transfection group; 7, 8 and 9, pcDNA3.1-TAp63 $\gamma$ transfection group; 9 , 10 and 11, p53 transfection group; 1 , 4, 7 and 10, cytosol protein; 2, 5, 8 and 11, mitochondrial protein; 3, 6, 9 and 12, nucleus protein. (B) Signals of AIF and Bit1 were analyzed in the subcellular fractions. a, untreated group; b, pcDNA3.1 transfection group; c, pcDNA3.1-TAp63 $\gamma$ transfection group; d, pcDNA3.1-p53 transfection group.
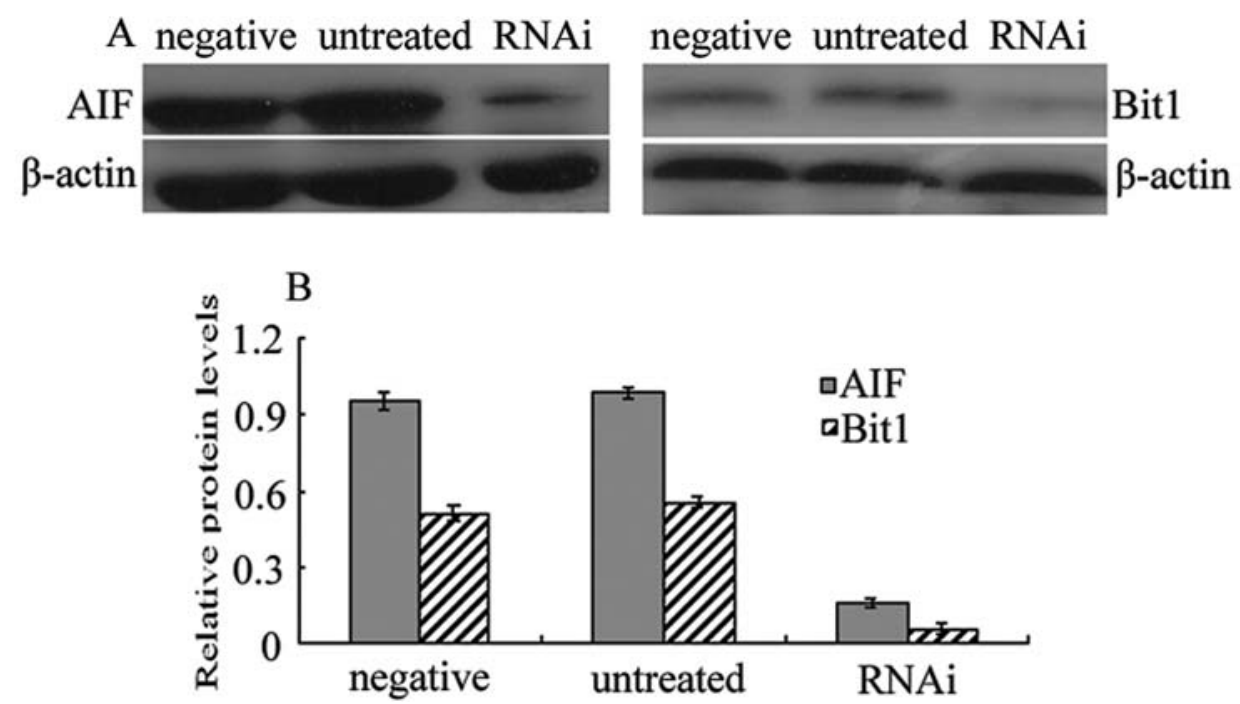

Figure 4. Effect of down-regulation of AIF or Bit1 protein by corresponding siRNA transfection. The densitometry data of AIF or Bit1/densitometry of $\beta$-actin $(\%)$ are the average of three independent experiments.

inhibited, but not completely blocked by the pan-caspase inhibitor z-VAD-fmk.

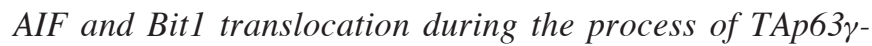
induced EC9706 cell apoptosis. After transfected with pcDNA3.1, pcDNA3.1-TAp63 $\gamma$ or pcDNA3.1-p53 $24 \mathrm{~h}$, respectively, the EC9706 cells were harvested. The nucleus, mitochondrial and cytosol fractions were extracted. As loading controls, a nuclear protein (histone), a mitochondrial protein (VDAC) and a cytosol protein (ß-actin) were used to examine the identity and purity of the the subcellular fractions. The results are show in Fig. 3A. Western blot assay to examine the subcellular location of AIF and Bit1 was also performed. AIF, in both nucleus and cytosol proteins, was detected in the cells transfected with either TAp63 $\gamma$ or p53 expression vector, but not in the cells transfected with pcDNA3.1 control (Fig. 3B). However, Bit1 protein could only be seen in the cytosol, not in the nuclei of the cells transfected with either TAp63 $\gamma$ or p53 expression vector (Fig. 3B). Either transfected with pcDNA3.1-TAp63 $\gamma$ or with pcDNA3.1-p53, AIF protein became gradually weaker in the mitochondria, and little by little stronger first in the cytosol and then in the nuclei, indicating that AIF protein was first translocated into the cytosol and then the nucleus while Bit1 was only translocated into the cytosol.

Down-regulation of AIF/Bit1 by RNAi can alleviate TAp63yinduced EC9706 cell apoptosis. Finally, to identify the relationships between AIF/Bit1 function and TAp63 $\gamma$-induced cell apoptosis, the EC9706 cells were transfected with 
Table I. The number of the positive apoptotic cells in different groups.

\begin{tabular}{|c|c|c|}
\hline Group & Cell no. & Positive apoptotic cell no. \\
\hline pcDNA3.1 & 1500 & 34 \\
\hline pcDNA3.1-TAp63 $\gamma$ & 1500 & $239^{\mathrm{a}}$ \\
\hline AIF-SiRNA+TAp63 $\gamma$ & 1500 & $93^{\mathrm{a}, \Delta}$ \\
\hline Bit1-SiRNA+TAp63 $\gamma$ & 1500 & $110^{\mathrm{a}, \Delta}$ \\
\hline
\end{tabular}

${ }^{a}$ denotes comparison with pcDNA3.1 transfection group, $\mathrm{P}<0.05 ;{ }^{\Delta}$ Refers to comparison with pcDNA3.1-TAp63 $\gamma$ transfection group, $\mathrm{P}<0.05$.
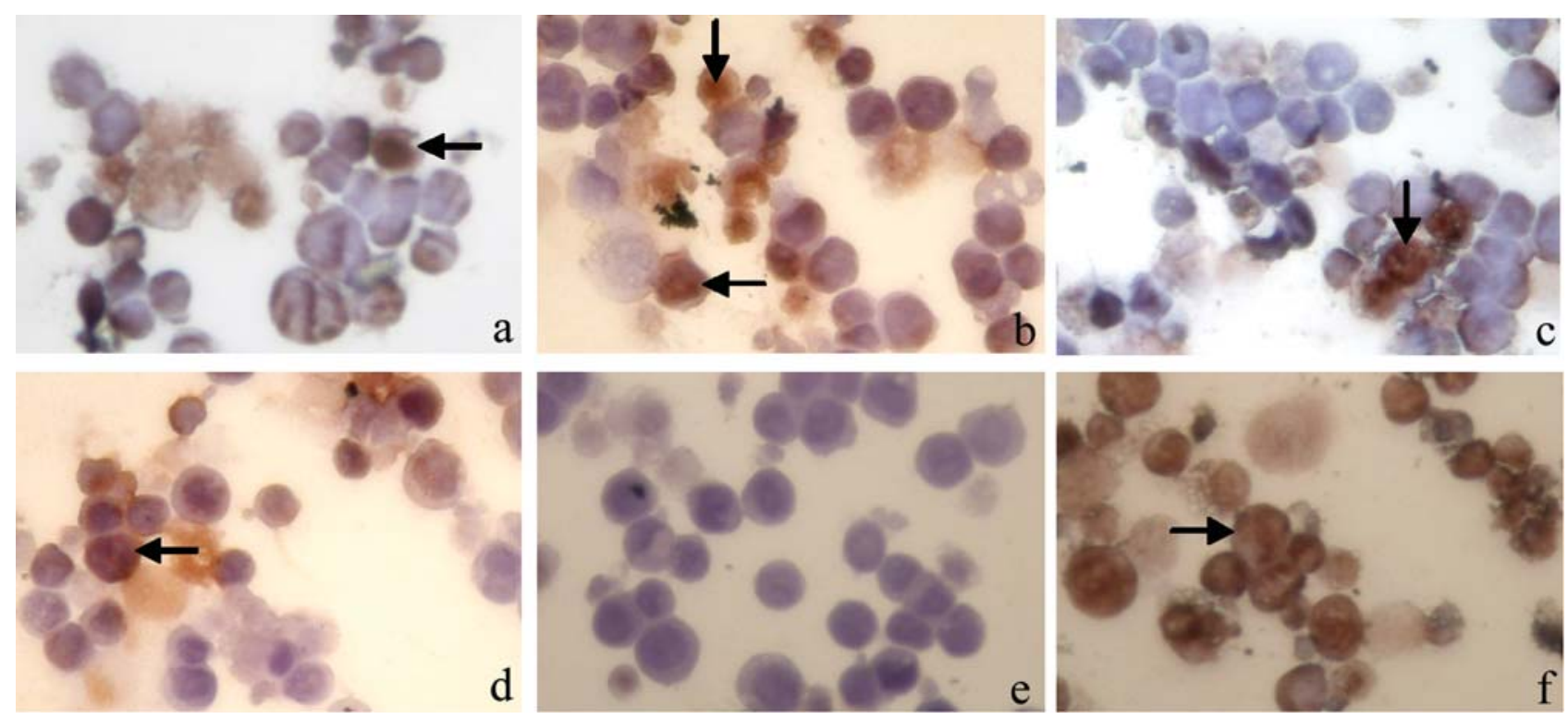

Figure 5. Detection of positive apoptotic cells with TUNEL method (magnification, x400). (a) pcDNA3.1 transfection group. (b) pcDNA3.1-TAp63 $\gamma$ transfection group. (c) AIF-siRNA-treated and pcDNA3.1-TAp63 $\gamma$ transfection group. (d) Bit1-siRNA-treated and pcDNA3.1-TAp63 $\gamma$ transfection group. (e) Negative control group. (f) Positive control group.

recombinant pSilencer AIF-siRNA and pSilencer Bit1siRNA, respectively. Plain vector was employed as a negative control. The medium containing $400 \mu \mathrm{g} / \mathrm{ml} \mathrm{G} 418$ was used and changed every 2 days. Two weeks after transfection, drug-resistant cells were selected and harvested for Western blot assay. AIF and Bit1 protein levels were markedly reduced in the cells transfected with corresponding recombinant pSilencer, while they remained unchanged in the cells transfected with negative plasmid (Fig. 4), implying that AIF-siRNA and Bit1-siRNA down-regulated AIF and Bit1 protein expression, respectively.

After cells were AIF-siRNA or Bit1-siRNA treated, the cells were transfected with TAp63 $\gamma$ vector. Twenty-four hours later, TUNEL assay and flow cytometry were both performed. In AIF-siRNA treated and TAp63 $\gamma$ transfection group and Bit1-siRNA treated and TAp63 $\gamma$ transfection one, the number of apoptotic cells was 93/1500 and 110/1500, respectively (Table I, Fig. 5). Both were less than that of the TAp63 $\gamma$ transfection group, but more than that of the pcDNA3.1 transfection group. Flow cytometry showed that $1.37 \%$ of the control group cells were apoptotic, while
$13.64 \%$ of the TAp63 $\gamma$ transfected cells were apoptotic. However, the AIF-siRNA treated and TAp63 $\gamma$ transfection, cells showed only $4.52 \%$ of cells with apoptotic changes (Fig. 6A-d) and the TAp63 $\gamma$ transfected, Bit1-siRNA-treated cells had only $5.72 \%$ cells with apotosis (Fig. 6A-e), indicating that down-regulation of either AIF or Bit1 alleviates TAp63 $\gamma$ induced EC9706 cell apoptosis. However, none of these two factors could completely inhibit the TAp63 $\gamma$-induced cell apoptosis in these cells.

\section{Discussion}

p63 protein localization and expression in the basal or progenitor cells of several epithelial tissues such as the epidermis, hair follicles, sweat glands, cervix, tongue, esophagus, mammary glands and prostate (23-25). p63 expression is lost as these cells migrate from the basal layer and become terminally differentiated cells. In human esophageal squamous cell carcinoma, however, elevated expression of p63 was $\Delta \mathrm{Np} 63$, but not TAp63. Expression of $\Delta$ Np63 and deficiency of TAp63 may play a significant role 

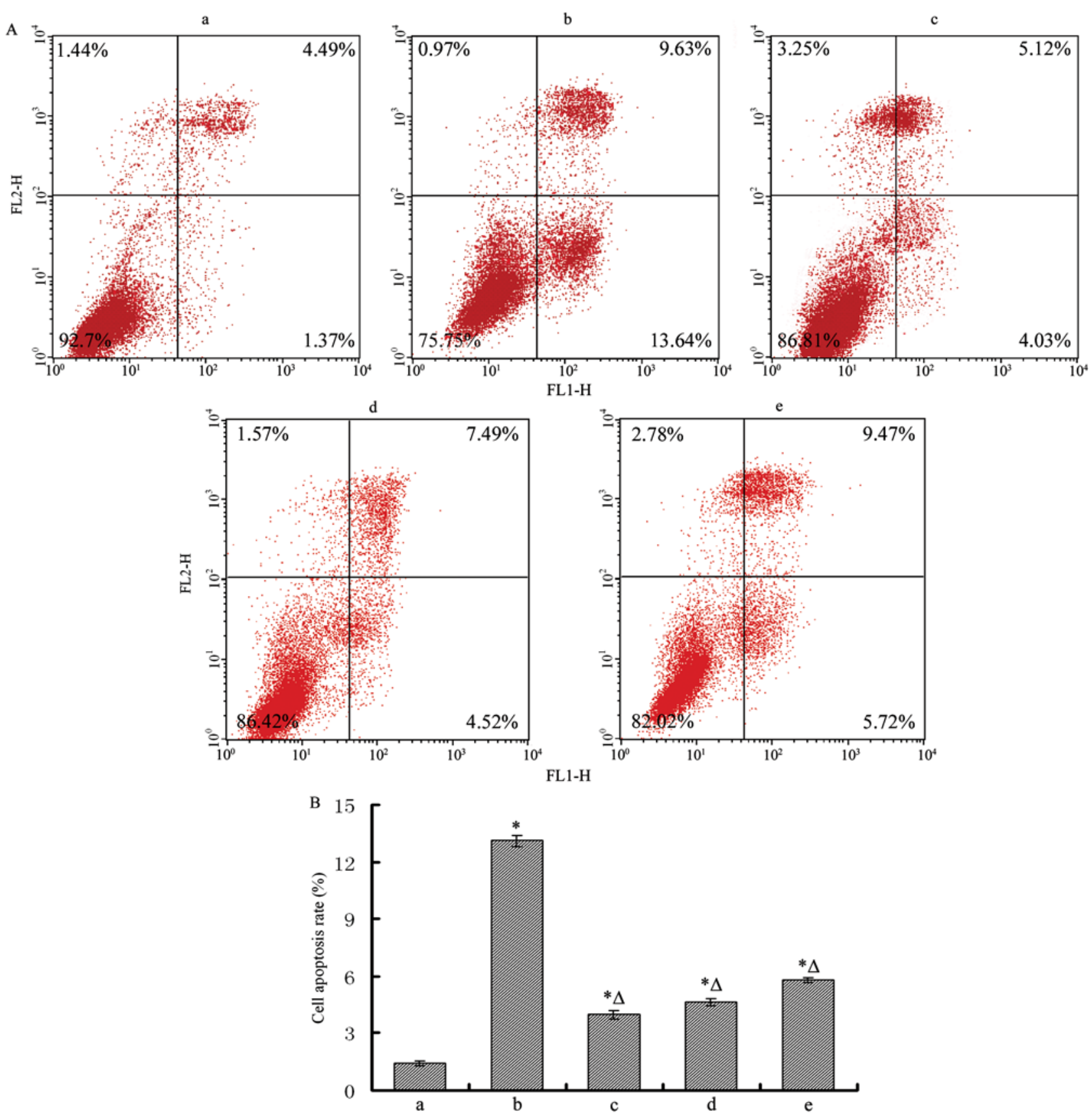

Figure 6. pcDNA3.1-TAp63 $\gamma$ transfection increased the number of Annexin V-positive cells $24 \mathrm{~h}$ after transfection and down-regulation of AIF or Bit1 can reduce TAp63 $\gamma$-induced EC9706 cell death. a, pcDNA3.1 transfection group; b, pcDNA3.1-TAp63 $\gamma$ transfection group; c, z-VAD-fmk treated and pcDNA3.1-TAp63 $\gamma$ transfection group; d, AIF-siRNA-treated and pcDNA3.1-TAp63 $\gamma$ transfection group; e, Bit1-siRNA-treated and pcDNA3.1-TAp63 $\gamma$ transfection group. The data are the apoptosis percentage of all groups and were obtained from three independent experiments. *denotes comparison with pcDNA3.1 transfection group $\mathrm{P}<0.05 ;{ }^{\Delta}$ refers to comparison with pcDNA3.1-TAp63 $\gamma$ transfection group $\mathrm{P}<0.05$.

in the development of this disease (26). Our results also verified the sub-type of p63 in EC9706 cells is $\triangle \mathrm{Np} 63$ only, not TAp63. It showed that both AIF and Bit1 were located in the cytoplasm of the cells.

We show here that TAp63 $\gamma$ can cause apoptosis through release of AIF and Bit 1 from mitochondria in the human esophageal cancer cell line EC9706. Both AIF and Bit1 contain a mitochondrial localization sequence $(20,21)$. Under physiological conditions, they are confined to the intermembrane space of mitochondria $(19,20)$. Under induction of apoptosis, they are released from mitochondria to participate in apoptotic process in a caspase-independent manner. We monitored cellular distribution of AIF and Bit1 during the TAp63 $\gamma$-induced apoptosis. However, differences exist. For example, Western blot analysis showed that EC9706 cells tranfected with TAp63 $\gamma$ expression vector resulted in the translocation of AIF from mitochondria to the nucleus via the cytosol. Only in the nucleus, AIF causes peripheral chromatin condensation and large scale DNA fragmentation $(20,27)$. Whereas Bit1 is released from mitochondria into cytosol, but not the nucleus, where it forms a complex with AES and promotes apoptosis. It has been shown that AIF 
translocation in response to p53-induced apoptosis signaling is abrogated in Bax-deficient neurons and transient overexpression of Bax was shown to trigger the release of AIF in $293 \mathrm{~T}$ and HeLa cells (16-18). It has also shown that overexpression of the antiapoptotic family member Bcl-2 prevents the release of AIF $(16,20)$. Similarly, cells expressing high levels of Bcl-2 or Bcl-xl may release too little endogenous Bit1 from their mitochondria to form apoptotic levels of Bit1/AES complexes (21). The mitochondrial localization and the manner of inducing apoptosis are very similar for AIF and Bit1 (28). Therefore, it would be interesting to know whether cells accept anoikis signals such as loss of attchment and AIF released from mitochondria and whether these two factors cooperate to promote caspase-independent forms of apoptosis.

TAp63 $\gamma$ expression causing release of AIF and Bit1 from mitochondria indicates TAp63 $\gamma$-induced apoptosis triggers the cell-intrinsic pathway. Bcl-2 family proteins whose members have both anti-apoptotic and pro-apoptotic activity have a central role in the control of mitochondria-induced apoptosis (29). It has been proposed that the Bcl-2 members form channels that facilitate protein transport and interaction with other mitochondrial proteins, and also induce rupture of the outer mitochondrial membrane (30). Both PUMA and NOXA, which encode the BH3 only domain proapoptotic protein of the $\mathrm{Bcl}$ protein family, are recently identified as p53 downstream target genes $(10,31)$. Induction of BH3-only Bcl-2 family members may contribute to the Bax translocation. Bax family members, a subfamily of Bcl-2 family proteins, act as tumor suppressors $(32,33)$. The presence of either Bax or Bak is essential for apoptosis in many cell types. They contribute to the permeabilization of the outer mitochondrial membrane, allowing efflux of apoptogenic proteins (34). While they regulate the mitochondrial pathway before caspase activation, the inhibitors of apoptosis proteins (IAP) regulate apoptosis after caspase activation (35).

In our study, interestingly, both down-regulation of AIF and that of Bit 1 can alleviate TAp63 $\gamma$-induced cell apoptosis, however, neither down-regulation of AIF nor that of Bit1 itself could completely inhibit it. Possibly during the process of TAp63 $\gamma$-induced apoptosis, other mitochondrial factors such as cyto-c and endonuclease $\mathrm{G}$ were released from mitochondria and involved in the initiation of the cellextrinsic apoptotic pathway as well. However, this is a matter of further study.

In summary, we report that TAp63 $\gamma$ can induce human esophageal squamous carcinoma cell apoptosis. It was discovered that AIF was released from mitochondria into nucleus via the cytosol but Bit1 was only released from mitochondria into cytosol. Furthermore, down-regulation of AIF and that of Bit1 by RNAi can alleviate TAp63 $\gamma$-induced cell apoptosis. Our results inducate that TAp63 $\gamma$-induced apoptosis can be mediated by AIF and Bit1 released from mitochondria in human esophageal cancer cell line EC9706. Thus, beyond TAp63 $\gamma$ 's importance in development and differentiation, we have elucidated that AIF and Bit1, which are both executors of the mitochondrial apoptosis pathway are, at least in part, responsible for the cell death effects of TAp63 $\gamma$ expression in esophageal squamous carcinoma cells.

\section{Acknowledgements}

We thank Dr Yaohe Wang for kindly providing vector containing the target gene. This study was supported by grants from Key Subject Program 'The Tenth Five-Year Plan' '211 Project' (Education Ministry of China, 2002-2) and was done in Henan Key Laboratory of Molecular Medicine.

\section{References}

1. Waltermann A, Kartasheva NN and Dobbelstein M: Differential regulation of p63 and p73 expression. Oncogene 22: 5686-5693, 2003.

2. Maisse C, Guerrieri P and Melino G: p73 and p63 protein stability: the way to regulate function? Biochem Pharmacol 66: 1555-1561, 2003.

3. Yang A, Kaghad M, Wang Y, et al: p63, a p53 homolog at 3q27-29, encodes multiple products with transactivating, deathinducing, and dominant-negative activities. Mol Cell 2: 305-316, 1998.

4. Bergamaschi D, Samuels Y, Jin B, Duraisingham S, Crook T and Lu X: ASPP1 and ASPP2: common activators of p53 family members. Mol Cell Biol 24: 1341-1350, 2004.

5. Osada M, Ohba M, Kawahara C, et al: Cloning and functional analysis of human $\mathrm{p} 51$, which structurally and functionally resembles p53. Nat Med 4: 839-843, 1998.

6. Attardi LD, Reczek EE, Cosmas C, Demicco EG, McCurrach ME, Lowe SW and Jacks T: PERP, an apoptosis-associated target of p53, is a novel member of the PMP-22/gas3 family. Genes Dev 14: 704-718, 2000.

7. Ihrie RA, Reczek E, Horner JS, Khachatrian L, Sage J, Jacks T and Attardi LD: Perp is a mediator of p53-dependent apoptosis in diverse cell types. Curr Biol 13: 1985-1990, 2003.

8. Ihrie RA, Marques MR, Nguyen BT, et al: Perp is a p63regulated gene essential for epithelial integrity. Cell 120: 843$856,2005$.

9. Ihrie RA and Attardi LD: A new perp in the lineup: linking p63 and desmosomal adhesion. Cell Cycle 4: 873-876, 2005.

10. Chipuk JE, Bouchier-Hayes L, Kuwana T, Newmeyer DD and Green DR: PUMA couples the nuclear and cytoplasmic proapoptotic function of p53. Science 309: 1732-1735, 2005.

11. Chipuk JE, Kuwana T, Bouchier-Hayes L, Droin NM, Newmeyer DD, Schuler M and Green DR: Direct activation of Bax by $\mathrm{p} 53$ mediates mitochondrial membrane permeabilization and apoptosis. Science 303: 1010-1014, 2004.

12. Rosse T, Olivier R, Monney L, et al: $\mathrm{Bcl}-2$ prolongs cell survival after Bax-induced release of cytochrome c. Nature 391: 496-499, 1998.

13. Tikhomirov O and Carpenter G: Bax activation and translocation to mitochondria mediate EGF-induced programmed cell death. J Cell Sci 118: 4681-4690, 2005.

14. Dohn M, Zhang S and Chen X: p63alpha and DeltaNp63alpha can induce cell cycle arrest and apoptosis and differentially regulate p53 target genes. Oncogene 20: 3193-3205, 2001.

15. Cregan SP, Dawson VL and Slack RS: Role of AIF in caspasedependent and caspase-independent cell death. Oncogene 23: 2785-2796, 2004.

16. Cregan SP, Fortin A, MacLaurin JG, et al: Apoptosis-inducing factor is involved in the regulation of caspase-independent neuronal cell death. J Cell Biol 158: 507-517, 2002.

17. Arnoult D, Parone P, Martinou JC, Antonsson B, Estaquier J and Ameisen JC: Mitochondrial release of apoptosis-inducing factor occurs downstream of cytochrome c release in response to several proapoptotic stimuli. J Cell Biol 159: 923-929, 2002.

18. Arnoult D, Gaume B, Karbowski M, Sharpe JC, Cecconi F and Youle RJ: Mitochondrial release of AIF and EndoG requires caspase activation downstream of $\mathrm{Bax} / \mathrm{Bak}$-mediated permeabilization. EMBO J 22: 4385-4399, 2003.

19. Wang X, Yang C, Chai J, Shi Y and Xue D: Mechanisms of AIF-mediated apoptotic DNA degradation in Caenorhabditis elegans. Science 298: 1587-1592, 2002.

20. Susin SA, Lorenzo HK, Zamzami N, et al: Molecular characterization of mitochondrial apoptosis-inducing factor. Nature 397: 441-446, 1999.

21. Jan Y, Matter M, Pai JT, et al: A mitochondrial protein, Bit1, mediates apoptosis regulated by integrins and Groucho/TLE corepressors. Cell 116: 751-762, 2004. 
22. Yuan CQ, Li YN and Zhang XF: Down-regulation of apoptosisinducing factor protein by RNA interference inhibits UVAinduced cell death. Biochem Biophys Res Commun 317: 11081113, 2004.

23. Levrero M, De laurenzi V, Costanzo A, Gong J, Wang JY and Melino G: The p53/p63/p73family of transcription factors: overlapping and distinct functions. J Cell Sci 113: 1661-1670, 2000.

24. Westfall MD, Mays DJ, Sniezek JC and Pietenpol JA: The Delta Np63 alpha phosphoprotein binds the p21 and 14-3-3 sigma promoters in vivo and has transcriptional repressor activity that is reduced by Hay-Wells syndrome-derived mutations. Mol Cell Biol 23: 2264-2276, 2003.

25. Di Como CJ, Urist MJ, Babayan I, et al: p63 expression profiles in human normal and tumor tissues. Clin Cancer Res 8: 494-501, 2002.

26. $\mathrm{Hu} \mathrm{H}, \mathrm{Xia} \mathrm{SH}, \mathrm{Li} \mathrm{AD}$, et al: Elevated expression of p63 protein in human esophageal squamous cell carcinomas. Int J Cancer 102: 580-583, 2002.

27. Lorenzo HK, Susin SA, Penninger J and Kroemer G: Apoptosis inducing factor (AIF): a phylogenetically old, caspaseindependent effector of cell death. Cell Death Differ 6: 516-524, 1999.
28. Stupack DG and Cheresh DA: A Bit-role for integrins in apoptosis. Nat Cell Biol 6: 388-389, 2004.

29. Cory S, Huang DC and Adams JM: The Bcl-2 family: roles in cell survival and oncogenesis. Oncogene 22: 8590-8607, 2003.

30. Hengartner MO: The biochemistry of apoptosis. Nature 407: 770-776, 2000.

31. Oda E, Ohki R, Murasawa H, et al: Noxa, a BH3-only member of the Bcl-2 family and candidate mediator of p53-induced apoptosis. Science 288: 1053-1058, 2000.

32. Willis SN and Adams JM: Life in the balance: how BH3-only proteins induce apoptosis. Curr Opin Cell Biol 17: 617-625, 2005.

33. Willis SN, Chen L, Dewson G, et al: Proapoptotic Bak is sequestered by Mcl-1 and Bcl-xL, but not Bcl-2, until displaced by BH3-only proteins. Genes Dev 19: 1294-1305, 2005.

34. Rampino N, Yamamoto H, Ionov Y, Li Y, Sawai H, Reed JC and Perucho M: Somatic frameshift mutations in the BAX gene in colon cancers of the microsatellite mutator phenotype. Science 275: 967-969, 1997.

35. Marsoni S and Damia G: Molecular targeting: new therapeutic strategies to improve tumour apoptosis. Ann Oncol 15: 229-231, 2004. 